\title{
Ultrathin liquid films under alternating intermolecular potential fields and capillary force
}

\author{
Kahp Y. Suh and Hong H. Lee ${ }^{\text {a) }}$ \\ School of Chemical Engineering and Nanoelectronics Institute, Seoul National University, Seoul, \\ 151-742, Korea
}

(Received 1 April 2002; accepted 2 July 2002)

A unique experimental system is devised that reveals the interplay among capillary force and alternating intermolecular forces. As a consequence of the interplay, untrathin $(<10 \mathrm{~nm})$ liquid films, which invariably experience dewetting, can be made stable, leading to a smooth and dropless minimum-potential surface. Theory and experiment show that the film thickness is $<1 \mathrm{~nm}$ when the film recedes in spite of the capillarity and it is $<3 \mathrm{~nm}$ when it rises into a cavity. (c) 2002 American Institute of Physics. [DOI: 10.1063/1.1502653]

\section{INTRODUCTION}

Thermal stability of thin liquid films on solid substrates has been extensively studied in recent years, both experimentally ${ }^{1-8}$ and theoretically ${ }^{9-13}$ due to its technological importance and scientific interest. The thermodynamic stability of the film is directly related to the sign of the second derivative of the free energy across the substrate-filmair system, which is given by ${ }^{14}$

$$
\Delta G=-\frac{A_{\text {eff }}}{12 \pi h_{0}^{2}},
$$

where $\Delta G$ is the excess free energy of the layer, $A_{\text {eff }}$ is the effective Hamaker constant for the van der Waals interactions of the film with the surrounding media, and $h_{0}$ is the initial film thickness. In some cases, $\partial^{2} \Delta G / \partial h^{2}$ is negative such that the film is unstable and ruptures, resulting in roughened or droplet structures on the surface.

There are two competing factors that dominate the thermodynamic and kinetic behavior of dewetting: One is the intermolecular forces, which activate the dewetting ( $A_{\text {eff }}$ $>0$ ) and the other the surface tension, which suppresses the dewetting. As a result, the dewetting process is determined by the balance between these two contributions, on which most of the literature have focused. ${ }^{1-13}$

Introducing capillarity into the picture has the significance of altering the balance between intermolecular forces and surface energy and of shifting the focus from undesirable dewetting to stable ultrathin film formation. Ordinarily, Laplace pressure $\left(\Pi_{L}\right)$ overwhelms disjoining pressure $\left(\Pi_{D}\right)$ when microcapillaries are used. For example, $\Pi_{L}$ is $10^{3}-10^{4}\left(\mathrm{~N} / \mathrm{m}^{2}\right)$ and $\Pi_{D}$ is $10^{1}-10^{2}\left(\mathrm{~N} / \mathrm{m}^{2}\right)$ for the film several tens of nanometers thick even when the cavity size is on the order of micrometer. Therefore, the film should be thinner than $10 \mathrm{~nm}$ to clearly elucidate the interplay between Laplace and disjoining pressures.

\footnotetext{
a) Author to whom correspondence should be addressed. Electronic mail: honghlee@plaza.snu.ac.kr
}

Another notable concept here is the introduction and realization of alternating intermolecular potential fields, which can be made competitive or cooperative although the competition is used in this work.

In this paper, we report a novel experimental method that can reveal the interplay among the capillary force and the intermolecular forces. Another unique feature of our experiment is the competition between two alternating intermolecular potential fields imposed on the liquid film. As a consequence of the interplay, a smooth and dropless surface layer forms that corresponds to the minimum of interfacial potential of the film instead of the common dewetting. To explain the rise or fall of the film in the cavity and the formation of the minimum-potential surface (MPS), we construct the effective potential for the film.

\section{EXPERIMENTAL METHOD}

For the experimental setup, we used a technique called "Capillary Force Lithography (CFL)." 15 When an elastomeric mold such as polydimethylsiloxane (PDMS) with a desired pattern is placed on a solid film surface and heated above the melting temperature, capillary force allows the liquid film to fill up the void space of the channels formed between the mold and the film, thereby generating a negative replica of the mold.

We fabricated PDMS (Sylgard 184, Dow Corning) molds that have a planar surface with recessed patterns by casting PDMS against a complementary relief structure prepared by photolithographic method. ${ }^{16}$ Line-and-space patterns $(150 \mathrm{~nm}-3 \mu \mathrm{m})$ with various step heights $(25-550 \mathrm{~nm})$ were used in our experiment. Silicon dioxide wafer that was used as a substrate was cleaned by ultrasonic treatment in trichloroethylene and methanol for 5 min each and dried in nitrogen. The substrate was then dipped in an octadecanethiol (ODT, $\left.\mathrm{CH}_{3}-\left(\mathrm{CH}_{2}\right)_{17}-\mathrm{SH}\right) /$ ethanol solution (0.1 $\mathrm{g} / 50 \mathrm{ml}$ ) for $5 \mathrm{~min}$ to $1 \mathrm{~h}$.

The mold with the pattern was placed on the surface of the as-formed ODT (Aldrich, 98\%) layer and then heated well above the melting temperature $\left(31-35^{\circ} \mathrm{C}\right)$ of ODT to $150{ }^{\circ} \mathrm{C}$ for $24 \mathrm{~h}$. Schematic diagram of the experimental pro- 

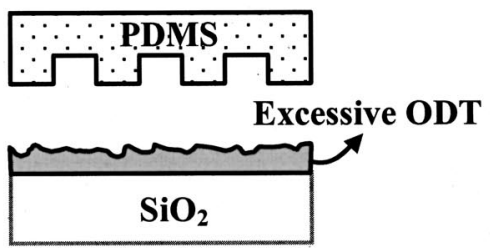

$\prod$ Mold contact and heating to $150^{\circ} \mathrm{C}$

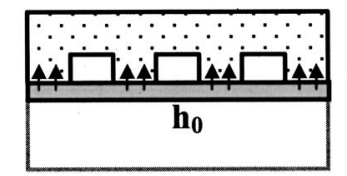

Absorption of ODT into the mold
$L$ is relatively small

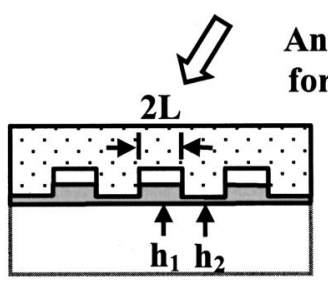

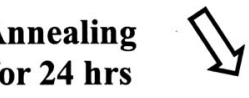

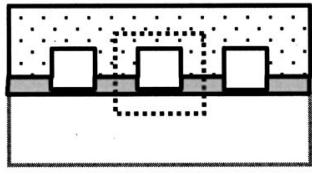

$L$ is relatively large (a)

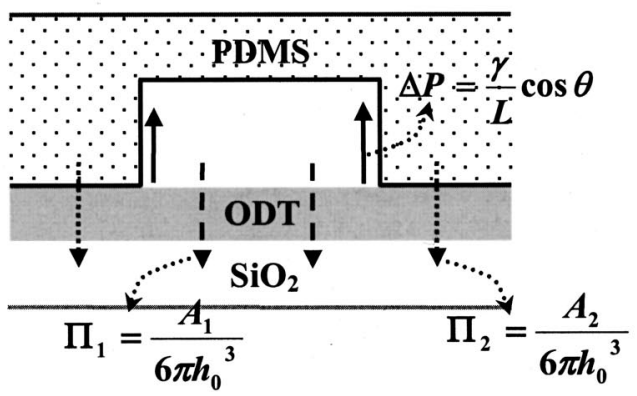

(b)

FIG. 1. (a) Schematic diagram of the experimental procedure where the thickness of the ODT layer is exaggerated for better illustration. Not that the film gets thinner and self-flattens due to the absorption of ODT into the mold. (b) Illustration of the capillary and intermolecular forces acting at the wall and through the multilayer [boxed square in Fig. 1(a)].

cedure is shown in Fig. 1(a). The film thickness was measured by elipsometry and confirmed by scratching experiment. The surface of the physisorbed ODT layer is quite rough. However, a considerable amount of ODT film is immediately absorbed into the PDMS mold when the mold is placed on the surface. As there are weak interactions between the substrate and the liquid film, an ultrathin film $(<10 \mathrm{~nm})$ always remains on the surface. It is noted that after the absorption, the film thickness decreases drastically and "selfflattens." To examine the surface structure, atomic force microscopy (AFM) measurements were made with a Nanoscope IIIa (Digital Instrument), operated in the tapping mode.

\section{RESULTS AND DISCUSSION}

In this study, we chose ODT as a liquid film because it is solid at room temperature and has a low melting temperature $\left(31-35^{\circ} \mathrm{C}\right)$, which helps analyze the surface at ambient environment. For comparison purposes, we have also applied the system to a low molecular weight polymer such as polystyrene $(\mathrm{MW}=3900)$ and observed similar results. With such a polymer, it is very difficult to prepare untrathin uniform films $(<10 \mathrm{~nm})$ on a large area and the polymer is very unstable along the channel direction, making it unsuitable for simple analysis. Although ODT has not been studied much in dewetting experiments, it is an inert liquid and has little chemical interactions with the $\mathrm{SiO}_{2}$ substrate.

Figure 2 shows typical morphologies of the dewetted patterns for an ODT film $3.7 \mathrm{~nm}$ thick when a flat mold is used. The pattern of the surface outside the area contacted with the mold is shown in Fig. 2(a), which results from isotropic dewetting and is clearly characterized by a single length-scale. The inset shows a Fourier transform of the image. The well-defined ring indicates the existence of a characteristic length-scale, while the radial symmetry of the transform confirms the isotropy of the dewetted pattern. A similar morphology is observed for the surface that is in contact with the mold in Fig. 2(b). In this case, the surface is a little rough and is characterized by a longer length scale than in Fig. 2(a) as shown by the Fourier transform image in the inset. The height [Fig. 2(c)] and phase [Fig. 2(d)] images of the scratched surface confirm the accuracy of the film thickness. From the results, we can conclude that the ODT layer is thermodynamically unstable with respect to the substrate and the PDMS mold.

Figure 3 shows the AFM images of a $3.7 \mathrm{~nm}$ ODT film for (a) $500 \mathrm{~nm}$ line/1200 nm space and (b) $150 \mathrm{~nm}$ line/650 $\mathrm{nm}$ space patterns. The depth of the mold cavities is $97 \mathrm{~nm}$ for (a) and $27 \mathrm{~nm}$ for (b), respectively. As shown in the figure, contrasting behavior is observed depending on the channel width. For a relatively large channel width $(500 \mathrm{~nm})$ shown in Fig. 3(a), the film under the void of the channel recedes, thus leading to a thickness difference of about 2.1 $\mathrm{nm}$ between the recessed region (valley) and the adjacent region that was in contact with the mold. Two separate menisci are localized at both walls of the void channel with a height of about $5.8 \mathrm{~nm}$ from the baseline. Therefore, the thickness of the film remaining on the valley region can be estimated to be about $0.9 \mathrm{~nm}$, which gives about $3.0 \mathrm{~nm}$ thick film on the region that was in contact with the mold.

To confirm the presence of ODT on the patterned surface, infrared spectroscopy (IR) measurements were made several times for the flat, adjacent regions contacted by the mold. After detecting three $\mathrm{CH}_{2}$ peaks at $2850-3000 \mathrm{~cm}^{-1}$, which is typical of ODT material, and analyzing the AFM cross-sectional profiles, we were led to the conclusion that ODT is present everywhere on the surface.

Due to the self-flattening by the PDMS mold, the roughness of the flat region is $<0.5 \mathrm{~nm}$, which is quite smaller than the reported value of $\sim 3 \mathrm{~nm}$ for octadecyltrichlorosilane (OTS) on a silicon oxide substrate annealed for $5 \mathrm{~h}$ at $150{ }^{\circ} \mathrm{C} .{ }^{17}$

On the other hand, the film fills the cavity of the mold to a thickness of about $5.4 \mathrm{~nm}$ for a relatively small channel width $(150 \mathrm{~nm})$ as shown in Fig. 3(b), which results from capillary rise. About $2.7 \mathrm{~nm}$ thick film remains on the sub- 

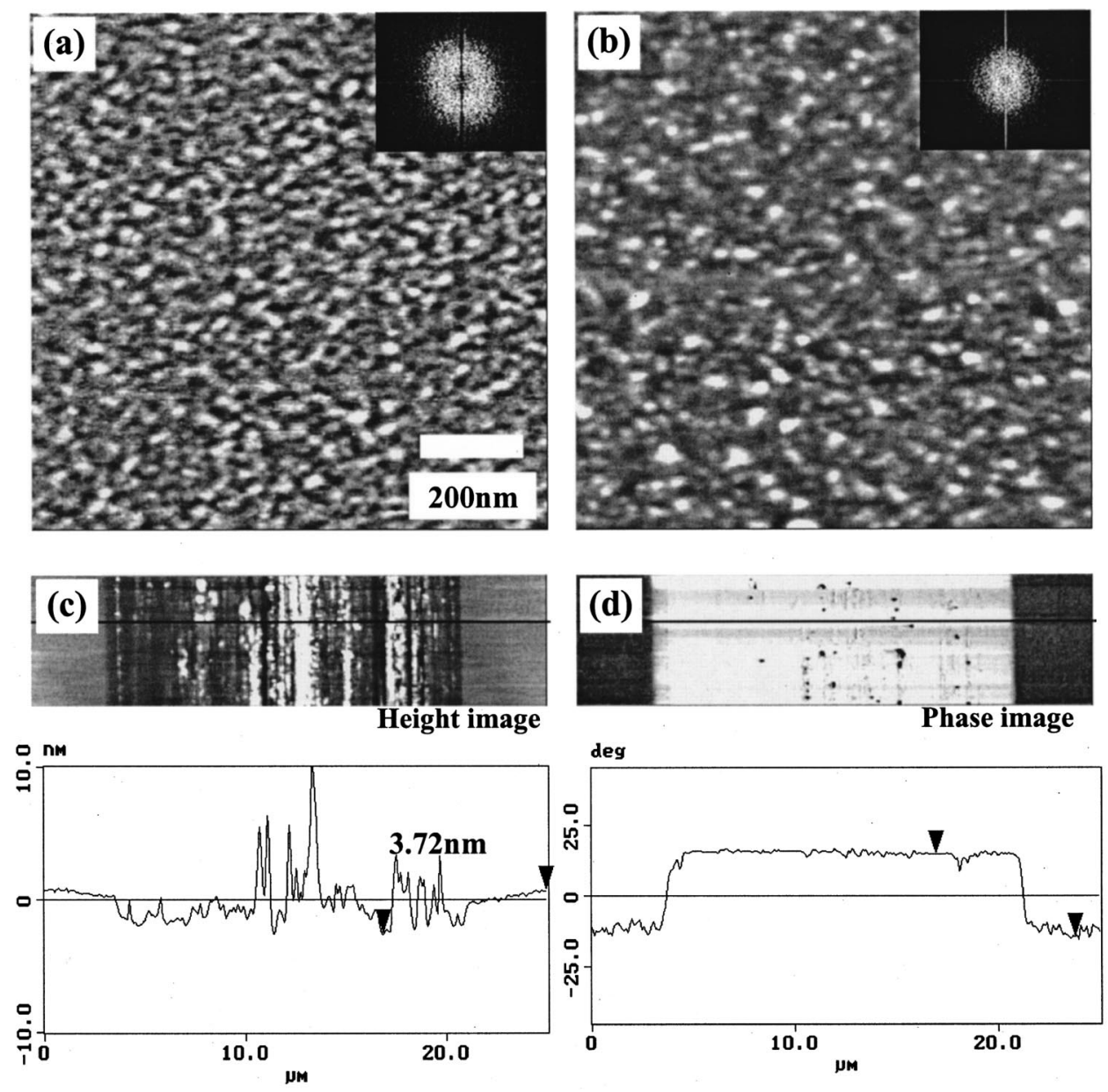

FIG. 2. Typical morphology $(1 \mu \mathrm{m}$ $\times 1 \mu \mathrm{m})$ of the dewetted pattern of the surface that is not in contact with the mold (a) and that in contact with the mold (b). A flat mold is used here. The height scale bar for (a) and (b) is $4 \mathrm{~nm}$. The inset shows Fourier transform images for each figure. The height (c) and phase (d) images of the scratched surface are also given to show film thickness. The samples were annealed at $150{ }^{\circ} \mathrm{C}$ for $24 \mathrm{hrs}$. strate under the regions that were in contact with the mold.

Figure 4 shows the relative height of the film within the channel with respect to the initial film thickness as a function of the channel width. All the values were obtained from the cross-sectional profiles of AFM images. The value of the relative height is negative when the film has receded towards the substrate. As seen in the figure, the behavior of the film clearly depends on the channel width. We did not observe the capillary rise for the channel width larger than about $1 \mu \mathrm{m}$, for which the film always recedes. This is due to the fact that it is very difficult to prepare thicker films $(\gg 10 \mathrm{~nm})$ because of absorption of the liquid into the mold in contact.

In short, our system can be viewed as a simple system in which two competing forces exist within the channel confinement: one is the Laplace pressure, or capillary force, the first derivative of which is negative so that it is stabilizing and the other is the intermolecular force, the first derivative of which is positive $\left(A_{\text {eff }}>0\right)$ so that it is destabilizing. The negative Laplace pressure is forced to equilibrate with the positive disjoining pressure, thus making the film stable even at the film thickness $<10 \mathrm{~nm}$.

Although our system is qualitatively well described by this simple picture, it is not clear how the two forces compete with each other quantitatively. In order to explain the behavior of the ultrathin liquid film in microcapillaries in more detail, we start with an effective free energy expression for the receding case in the unit structure shown in Fig. 1(b).
The energy can be written as the sum of two components for the line and the space,

$$
\begin{aligned}
\phi=\phi_{1}+n \phi_{2}= & \frac{C_{1}}{h_{1}^{8}}-\frac{A_{1}}{12 \pi h_{1}^{2}}-\frac{\Delta h}{L} \gamma \cos \theta \\
& +n\left(\frac{C_{2}}{h_{2}^{8}}-\frac{A_{2}}{12 \pi h_{2}^{2}}\right),
\end{aligned}
$$

where $n$ is the ratio of the width of the space to that of the line, $h_{1}\left(A_{1}\right)$ and $h_{2}\left(A_{2}\right)$ are the film thicknesses (Hamaker constants) under the channel and the space [see Fig. 1(a)], $C_{i}^{\prime}$ $(i=1,2)$ are the corresponding repulsion constants, respectively, $\Delta h\left(=h_{w}-h_{0}\right)$ is the capillary rise, $h_{w}$ being the meniscus height at the wall, $L$ is the half-channel width, $\gamma$ is the surface tension of the liquid, and $\theta$ is the contact angle. Instead of the exponential repulsion term, we chose a Lennard-Jones-type repulsion term. ${ }^{8}$ As the Hamaker constant for the layered structure is readily given by the individual Hamaker constants by mixing rule, there results ${ }^{18}$

$$
\begin{aligned}
& A_{1}=\left(\sqrt{A_{\mathrm{SiO}_{2}}}-\sqrt{A_{\mathrm{ODT}}}\right)\left(\sqrt{A_{\mathrm{air}}}-\sqrt{A_{\mathrm{ODT}}}\right), \\
& A_{2}=\left(\sqrt{A_{\mathrm{SiO}_{2}}}-\sqrt{A_{\mathrm{ODT}}}\right)\left(\sqrt{A_{\mathrm{PDMS}}}-\sqrt{A_{\mathrm{ODT}}}\right) .
\end{aligned}
$$

From the literature, $A_{\mathrm{SiO}_{2}}=2.2 \times 10^{-20}(\mathrm{~J}),{ }^{8,18} A_{\mathrm{ODT}}=6.0$ $\times 10^{-20}(\mathrm{~J}),{ }^{18} A_{\mathrm{PDMS}}=5.2 \times 10^{-20}(\mathrm{~J}),{ }^{19}$ and $A_{\text {air }}=0$, which gives $A_{1}=2.37 \times 10^{-20}(\mathrm{~J})$ and $A_{2}=1.53 \times 10^{-21}(\mathrm{~J})$. From 

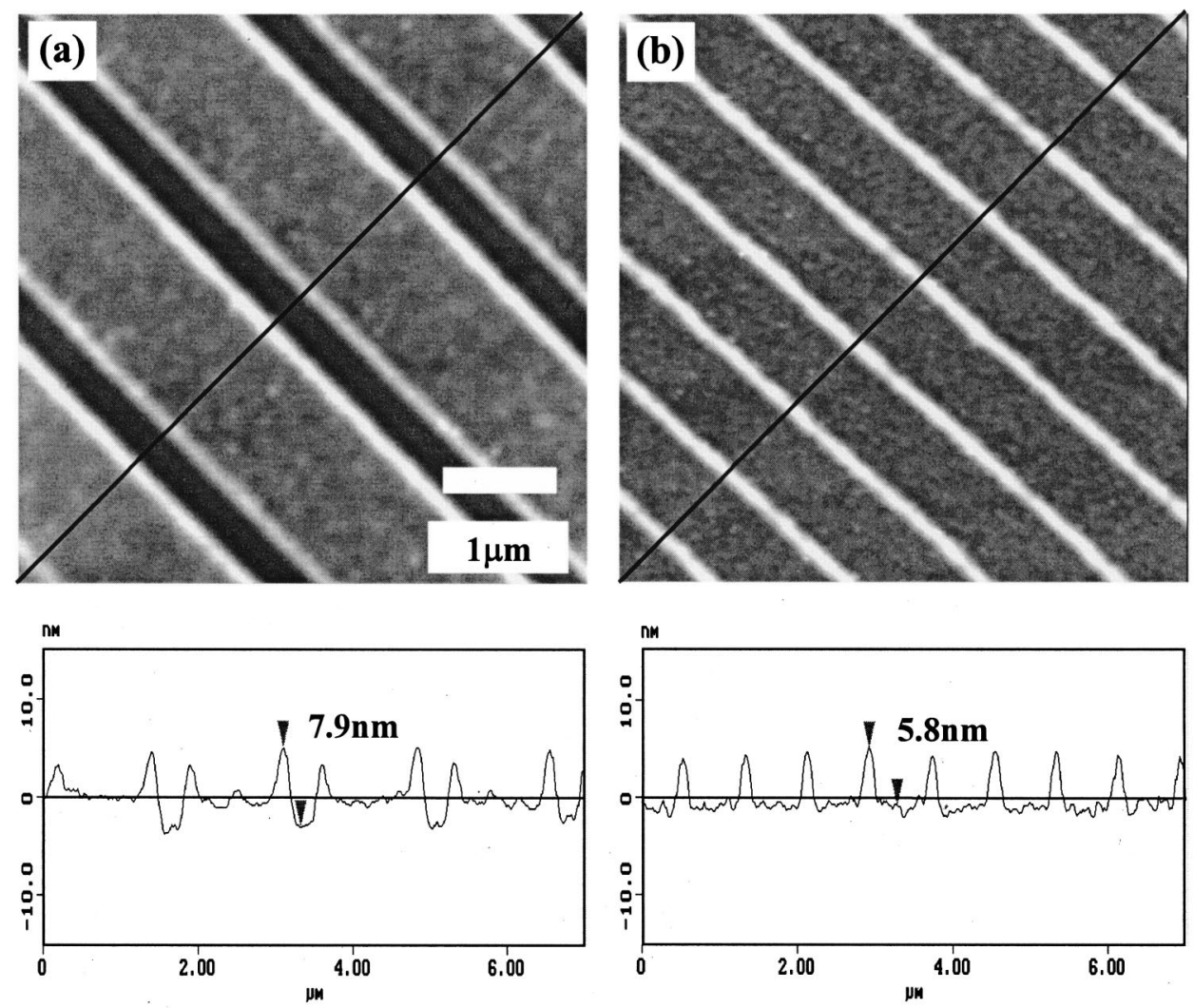

FIG. 3. Planar $(5 \mu \mathrm{m} \times 5 \mu \mathrm{m})$ and cross-sectional views of the AFM images for (a) $500 \mathrm{~nm}$ line $/ 1200 \mathrm{~nm}$ space and (b) $150 \mathrm{~nm}$ line/650 $\mathrm{nm}$ space patterns. The solid line in the figure indicates the original film height. The samples were prepared by the same conditions as in Fig. 2. the cross-sectional images in Fig. 3(a) and those for larger channel widths, the contact angle at the ODT-PDMS interface can be estimated to be about $85^{\circ}$. To determine the values of $C_{i}, \phi_{i}(i=1,2)$ can be minimized with respect to $h_{i}$. Then the values of $C_{i}$ can be obtained from the resulting equations with the experimentally measured values of $h_{1, \min }$ in Fig. $3\left(h_{1, \min }=0.9 \mathrm{~nm}\right.$ and $\left.h_{2, \min }=3.0 \mathrm{~nm}\right)$. The values thus obtained are $C_{1}=8.35 \times 10^{-77}\left(\mathrm{~J} \mathrm{~m}^{6}\right)$ and $C_{2}=7.4$ $\times 10^{-75}\left(\mathrm{~J} \mathrm{~m}^{6}\right)$.

For the rising case, the free energy can be written in a similar form as in Eq. (2) except that $\Delta h$ is $h_{1}-h_{0}$. The thickness of the film under the void of the mold, $h_{1}$, and that of the film in contact with the mold, $h_{2}$, as effected by the

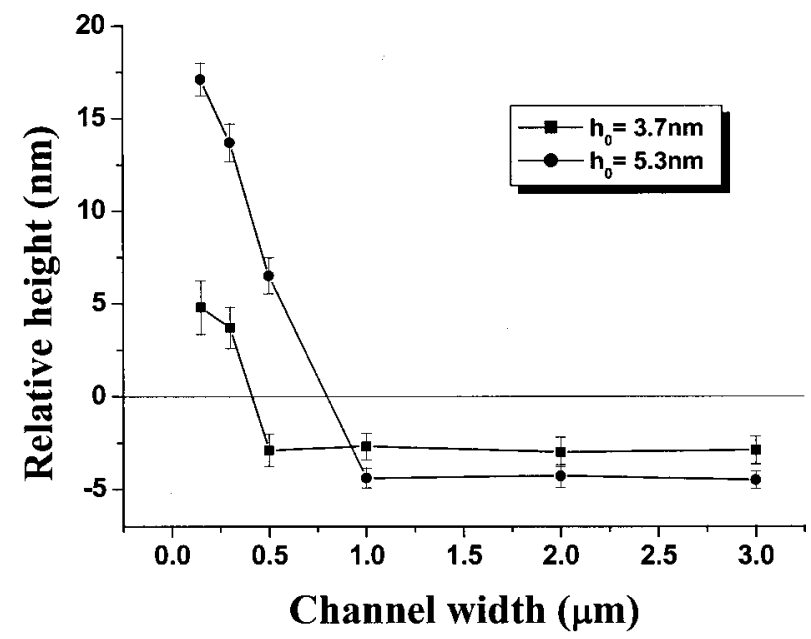

FIG. 4. Relative height of the film within the channel with respect to the initial film thickness as a function of the channel width. The value is negative when the film has receded towards the substrate. capillarity can be determined from Eq. (2) with the aid of the following mass balance for the unit structure of interest [Fig. 1(b)]:

$$
h_{1}+n h_{2}=(n+1) h_{0} .
$$

Minimization with respect to $h_{2}$, noting that $\partial h_{1}+n \partial h_{2}$ $=0$, gives

$$
\frac{\gamma}{L} \cos \theta=\frac{A_{1}}{6 \pi h_{1}^{3}}-\frac{A_{2}}{6 \pi h_{2}^{3}}+\frac{8 C_{2}}{h_{2}^{9}} .
$$

In Eq. (6), we neglect the repulsion term in $\phi_{1}$ since $h_{1}$ is considerably larger than $h_{2}$ due to capillarity. Shown in Fig. 5 are the plots of Eq. (6) rearranged with Eq. (5) for the solution of the equilibrium height $h_{2}$ with $C_{2}$ determined earlier. The plots are given as a function of the initial film thickness $h_{0}(5.3,6.5$, and $7.7 \mathrm{~nm})$ for the $500 \mathrm{~nm}$ line/1200 nm space pattern $(n=2.4)$. As apparent from Fig. 5, there are two solutions of $h_{2}$. However, one of the two solutions gives a value higher than the initial film thickness, which is physically unrealistic, yielding only one solution for $h_{2}$. Shown in the inset of the figure is the comparison between the predicted maximum capillary rise $\left(h_{1}-h_{0}\right)$, and the experimental values for various initial film thicknesses. The comparison shows that the predicted maximum height is in satisfactory agreement with the observed value. According to the theory, the equilibrium height of $h_{2}\left(h_{2, \min }\right)$ is about 2.5 $\mathrm{nm}$ regardless of the initial film thickness and the channel width (see Fig. 5). However, our experiment shows that a film layer of 2.5-2.9 $\mathrm{nm}$ remains on the surface depending on the individual experimental conditions. Considering the nonidealities that may exist in the experiment, the predicted value of $h_{2, \text { min }}$ is in good agreement with the experimental data. 


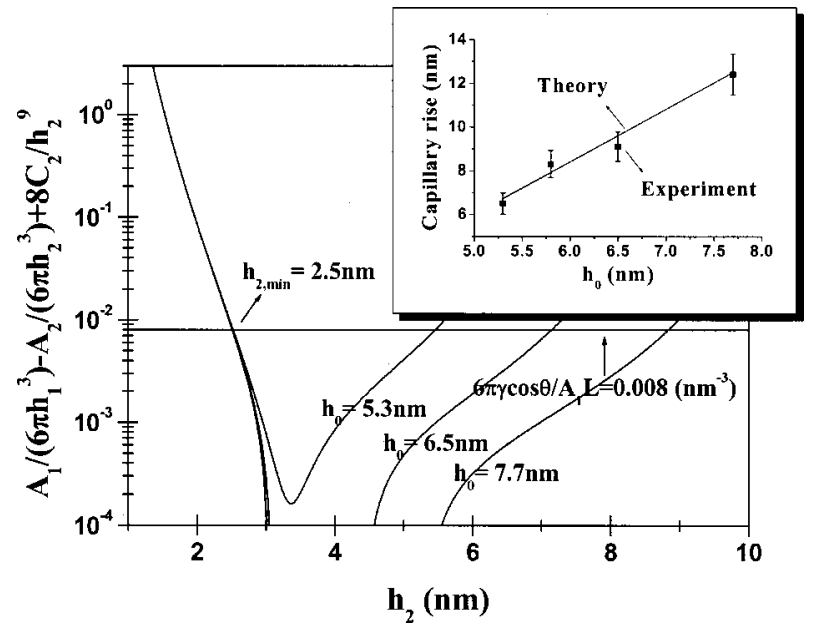

FIG. 5. Determination of the equilibrium film thickness, $h_{2}\left(h_{2, \min }\right)$, for the $500 \mathrm{~nm}$ line/1200 nm space pattern for three initial film thicknesses $\left(h_{0}\right.$ $=5.3,6.5$, and $7.7 \mathrm{~nm}$ ) when the film rises into the cavity. The inset compares the height of the capillary rise $\left(h_{1}-h_{0}\right)$ between the theory and the experiment.

One notable finding here is that a smooth and dropless MPS forms in the channel region for the recessed case as shown in Fig. 3(a). Without the capillarity, the conventional spinodal morphology would result as shown in Fig. 2(a). We attribute the origin to the interplay among the Laplace and intermolecular forces. As the capillarity acts as a mass sink in drawing mass from the surface, drops do not form and spinodal-like perturbations are suppressed. Once the MPS is completed, the force balance between the Laplace and intermolecular contributions makes the system very stable, thus leading to the flat structure under the channel. While there has been no result reported on the formation of ultrathin liquid films that are stable, there have been several studies reported for the stability of thin polymer films, involving the use of grafting ${ }^{20-22}$ and nanoparticles. ${ }^{23}$ However, the film thickness is on the order of $100 \mathrm{~nm}$, whereas the thickness of the stable film in this study is on the order of $1 \mathrm{~nm}$.

Another notable finding is that the capillary force modifies the interfacial potential. Figure 6 shows the potentials for the PDMS/ODT/ $/ \mathrm{SiO}_{2}$ structure (without capillarity) and for the unit structure shown in Fig. 1(b) (with capillarity) as a function of $h_{2}$. When the capillarity is not involved, the film thickness corresponding to the minimum potential is $3.0 \mathrm{~nm}$ as shown in Fig. 3(b). On the other hand, the value decreases to $2.5 \mathrm{~nm}$ when the capillarity comes into play. In other words, the capillarity plays a role as an attraction term, resulting in an effective decrease in the thickness of the minimum-potential layer.

\section{SUMMARY}

We have shown for the first time that ultrathin $(<10 \mathrm{~nm})$ liquid films can be made stable even at temperatures much higher than the melting temperature by introducing Laplace pressure. With the capillarity providing a mass sink, the balance between the Laplace pressure and the intermolecular forces makes the system stable, leading to a smooth and dropless surface. The alternating intermolecular potential

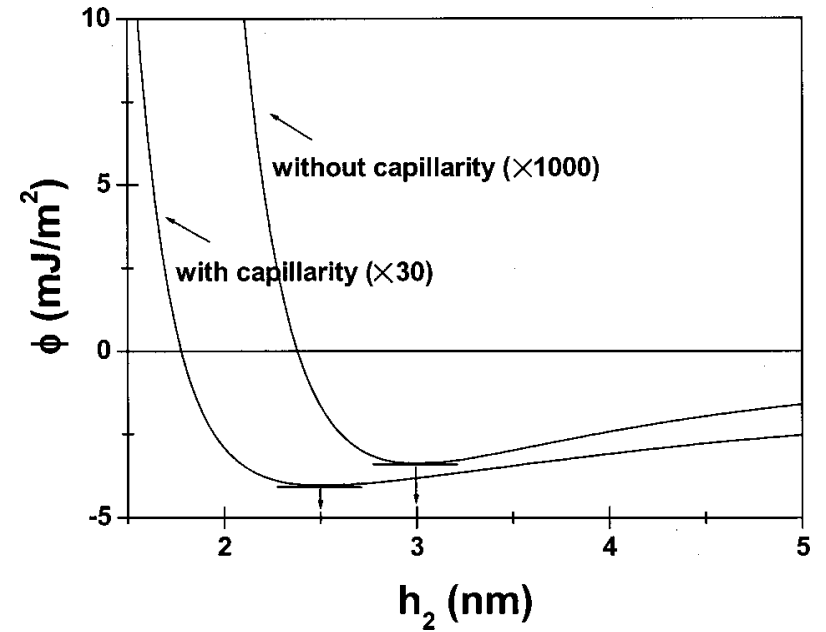

FIG. 6. Potential diagram as a function of $h_{2}$ for the PDMS/ODT/SiO structure (without capillarity) and for the unit structure shown in Fig. 1(b) (with capillarity). Note that $h_{2, \min }$ shifts from 3.0 to $2.5 \mathrm{~nm}$ due to the action of capillarity.

fields drive the film thickness down to a level corresponding to the minimum of the interfacial potential of the film. We have constructed effective potential diagrams when two alternating intermolecular forces come into play. It is shown that the capillarity gives rise to an effective decrease in the thickness of MPS. As a result, sub-1 nm films can be made that is stable and smooth. This result would open the door to utilization of pseudo physical "monolayer" as opposed to the chemical monolayer that has been the centerpiece for self-assembly since the physical monolayer can also have a functional group at the end of the molecule.

${ }^{1}$ G. Reiter, Phys. Rev. Lett. 68, 75 (1992).

${ }^{2}$ A. Milchev and K. Binder, J. Chem. Phys. 114, 8610 (2001).

${ }^{3}$ K. Jacobs, S. Herminghuas, and K. R. Mecke, Langmuir 14, 965 (1998).

${ }^{4}$ R. Xie, A. Karim, J. F. Douglas, C. C. Han, and R. A. Weiss, Phys. Rev. Lett. 81, 1251 (1998).

${ }^{5}$ K. Y. Suh and H. H. Lee, J. Chem. Phys. 115, 8204 (2001).

${ }^{6}$ M. Sferrazza, M. Heppenstall-Butler, R. Cubitt, D. Bucknall, J. Webster, and R. A. L. Jones, Phys. Rev. Lett. 81, 5173 (1998).

${ }^{7}$ G. Reiter and R. Khanna, Phys. Rev. Lett. 85, 2753 (2000).

${ }^{8}$ R. Seemann, S. Herminghaus, and K. Jacobs, Phys. Rev. Lett. 86, 5534 (2001)

${ }^{9}$ A. Vrij, Discuss, Faraday Soc. 42, 23 (1966).

${ }^{10}$ F. Brochard and J. Daillant, Can. J. Phys. 68, 1084 (1990).

${ }^{11}$ A. Sharma and R. Khanna, Phys. Rev. Lett. 81, 3463 (1998).

${ }^{12}$ J. Koplik and J. R. Banavar, Phys. Rev. Lett. 84, 4401 (2000).

${ }^{13}$ K. Y. Suh and H. H. Lee, Phys. Rev. Lett. 87, 135502 (2001).

${ }^{14}$ I. B. Ivanov, Thin Liquid Films: Fundamentals and Applications (Marcel Dekker, New York, 1988).

${ }^{15}$ K. Y. Suh, Y. S. Kim, and H. H. Lee, Adv. Mater. 13, 1386 (2001).

${ }^{16}$ Y. Xia, J. A. Rogers, K. E. Paul, and G. M. Whitesides, Chem. Rev. 99, 1823 (1999).

${ }^{17}$ M. Calistri-Yeh, E. J. Kramer, R. Sharma, W. Zao, M. H. Rafailovich, J. Sokolov, and J. D. Brock, Langmuir 12, 2747 (1996).

${ }^{18} \mathrm{~J}$. Israellachvili, Intramolecular Surfaces Forces, 2nd ed. (Academic, New York, 1992).

${ }^{19}$ M. O. David, G. Reiter, T. Sitthai, and J. Schultz, Langmuir 14, 5667 (1998).

${ }^{20}$ R. Yerushalmi-Rozen, J. Klein, and J. Fetters, Science 263, 793 (1994).

${ }^{21}$ Y. Feng, A. Karim, R. A. Weiss, J. F. Douglas, and C. C. Han, Macromolecules 31, 484 (1998).

${ }^{22}$ G. Henn, D. G. Bucknall, M. Stamm, P. Vanhoorne, and R. Jerome, Macromolecules 29, 4305 (1996).

${ }^{23}$ K. A. Barnes, A. Karim, J. F. Douglas, A. I. Nakatani, H. Gruell, and E. J. Amis, Macromolecules 33, 4177 (2000). 